\title{
Téoros
}

Revue de recherche en tourisme

\section{Marqué à vie !}

\section{André Hut}

Volume 20, numéro 1, printemps 2001

URI : https://id.erudit.org/iderudit/1071899ar

DOI : https://doi.org/10.7202/1071899ar

Aller au sommaire du numéro

\section{Éditeur(s)}

Université du Québec à Montréal

\section{ISSN}

0712-8657 (imprimé)

1923-2705 (numérique)

Découvrir la revue

\section{Citer ce document}

Hut, A. (2001). Marqué à vie ! Téoros, 20(1), 75-80.

https://doi.org/10.7202/1071899ar d'utilisation que vous pouvez consulter en ligne.

https://apropos.erudit.org/fr/usagers/politique-dutilisation/ 


\section{Témo ignage}

\section{Marqué à vie !}

\section{André Hut}

Ma récente retraite professionnelle a déclenché une nécessaire * re-précision $*$ de mes objectifs de vie en raison d'un temps a présent LIBRE d'être façonné à l'aune de mes désirs et de mes centres d'interêts personnels.

$\mathbf{L}$ 'éventualité d'un déménagement a commandé la mise en ordre de la documentation accumulée et la cession prochaine, à une faculté d'histoire universitaire, d'une bibliothèque assez unique sur l'évolution économique, culturelle et idéologique des pratiques touristiques pendant les quarante dernières années.

A cette occasion, que de souvenirs réveillés par certains auteurs fétiches, certains colloques ou numéros spéciaux de revues, considérés comme des jalons déterminants de l'histoire du tourisme. Quelle émotion à la lecture de certains textes, rédigés fiévreusement souvent de nuit, au fil des événements, subis ou provoqués, au cours de quatre décennies d"engagement militant et d'initiatives socioculturelles aussi audacieuses qu'inédites.

Les fréquentes et apaisantes promenades méditatives et solitaires le long du littoral belge, à La Panne, durant les séjours de repos médical, en hiver depuis dix ans, et celles, ensoleillées et lumineuses, sur la Côte d'Argent de l'océan Atlantique, au cours des vacances d'automne, à Mimizan... ont laissé progressivement émerger la prise de conscience, comme un reflux, de l'influence décisive que la mer a exercé, tant sur la construction en profondeur de ma personnalité que, sans aucun doute quoique inconsciemment, sur les décisions concernant les étapes de mon itinéraire professionnel.

Quel inattendu concours de circonstances que l'annonce du thème de la présente production de Téoros invitant justement à cerner les liens qu'entretiennent dialectiquement entre eux la mer et le tourisme !

Revenu de ma surprise, j'ai proposé d'évoquer mon vécu et de le resituer dans le contexte de la thématique choisie, notamment grâce aux notes (Hut, Boyer, Mucci, 1985).

\section{Thérapie}

À l'âge de 7 ans, mes parents et la faculté médicale m'ont envoyé au Préventorium Marin de Coq-sur-Mer oủ ma sæur, sujette à des crises d'asthme, sçjournait déjà. En effet, j'étais reconnu de faible constitution, surtout pulmonaire, et mal armé pour résister à la grave épidémie de tuberculose qui sévissait dans les pays européens durant l'entre-deux-guerres !

Quelle extraordinaire aventure pour un enfant de la capitale que de vivre pendant six mois, de septembre 1939 à février 1940 , au rythme des marées ! De fréquenter journellement la plage et les dunes désertes, successivement bercé par la douceur de l'automnal été indien, affronté aux violentes tempêtes de solstice, surpris par d'abondantes chutes de neige et la dureté de monticules d'écume pétrifiée par de fortes gelées ; de jouer avec les mouettes et de courir après les lapins, de s'écorcher aux piquants des épineux ou de se couper les doigts aux tiges des oyats; de s'inquiéter des brouillards épais et fantasmagoriques ou de s'extasier devant des couchers de soleil apocalyptiques!

A longueur de mois, supporter jusqu' à la lassitude le ronflement des vagues en colère, le hurlement des vents mugissants, le

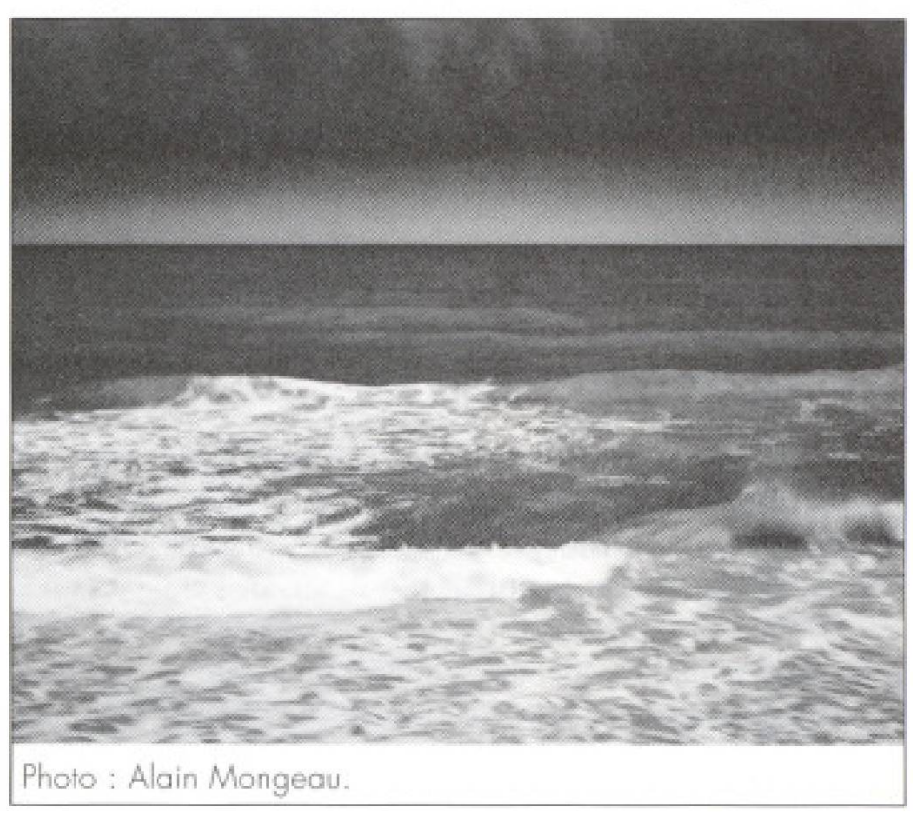


harcèlement des cris moqueurs des mouettes rieuses et le hululement lugubre des cornes de brume par lesquelles les pecheur's, dans leur barque, s"avertissent mutuellement de leur présence. Comment passer sous silence le florilège d'odeurs enregistrées - et aujourd'hui disparues -, subtilement différentes selon la direction du vent et l'endroit où l'on se trouve, sur la plage dégagée par la marée, proche des vagues, ou au sommet des hautes dunes, que l'on renifle les algues abandonnées sur la grève ou le crabe se débattant entre les doigts qui le tiennent prisonnier...

Il est certain que ces expériences multisensorielles et ces émotions primaires ont dû investir de manière déterminante et cumulative l'IMAGINAIRE de ce petit garçon de la ville particulièrement réceptif à cette période spécifique de l'enfance appelée « poétique $\%$.

C'est peut-être la raison de cet engouement ultérieur, en fin d'école primaire, pour la lecture boulimique de nombreux récits d'aventures, dont surtout les Vingt mille lienes sous les mers de Jules Verne, afin de retrouver les vibrations, en écho, de ces émotions enfouies et le sel des saveurs océanes (Corbin, 1988).

Le krach financier et la crise économique des années 1930 n'ont fait qu'accentuer la malnutrition et la vulnérabilité de la progéniture des parents ouvrier's, regroupés dans les insalubres régions industrielles ou les quartiers populaires des grandes villes. La nécessaire prévention en faveur de ces enfants à risque explique la création et la multiplication de centres de séjours permanents et de vacances, échelonnés le long du littoral, à l'initiative des écoles publiques ou confessionnelles, des organisations féminines socialistes ou chrétiennes, des institutions de santé publique comme l'Oeuvre Nationale de l'Enfance (ONE) (Hut, 1991).

Ces établissements issus du mouvement associatif privé ou public appartiennent désormais à l'archéologie touristique. Plutôt que de les démolir pour cause de vétusté et d'insecurité tout en réalisant par ailleurs de bénéfiques quoique paradoxales opérations immobilières et financières, à partir de ces localisations uniques et irremplaçables, ne faudrait-il pas en sauver l'un ou l'autre, par exemple en le réaffectant comme Centre de recherche et d'interprétation, pour ne pas laisser s'enliser dans les sables de l'histoire cette inédite entreprise du tourisme social et populaire autant qu'un des rares échantillons encore existants de cette spécilique architecture balnéaire?

D'ailleurs, cette dimension thérapeutique du tourisme côtier n'est pas une exception, mais bien un élément fondateur ${ }^{1}$ du tourisme. En effet, la classe aristocratique a suivi les conseils des médecins en transférant sur le littoral les séjour's de cure qu'ils fréquentaient dans les stations thermales de Spa, de Baden ou de Bath afin, selon la mode, de s'adonner aux bains de mer dans les stations balnéaires, cette fois, de la façade atlantique, durant l'été (Désert, 1983), a Les guides et traités du XIX siècle les assimilent au thermalisme ; c'est la valeur thérapeutique de la balnéation qui compte ; il n'est guère question de nage ou jeux de plage, encore moins de bains de soleil $\$$ (Boyer, $1982: 138$ ). Il en reste d'ailleurs des traces reperables sur certaines facades, en ce qui concerne les appellations : « Hôtel des Bains * ou * Kuursal *, comme aussi dans le nom composé des stations touristiques crées en bordures de plages qui comprennent le suffixe, en flamand, Bad (bain) alors que pour la commune d'origine, en recul géographique, c'est Dorp (village).

Mais vu le rythme saisonnier qui caractérise ce tourisme aristocratique, il ne s'agissait pas cependant d'abandonner l' habitude, prépondérante et plus ancienne, des longs séjours d'hivernants sur la Méditerranée.

Toute une litếrature médicale spécialisée tratte de l'usage de l'hiver dans le Midi' ${ }^{2}$ La température, les vents, l'hygrométrie, autant d'elements qu'il faut soigneusement peser et qui font successivement la renommé de telle ou telle station. La phisie d'abord mais aussi le lymphatisme, la langueur et autres maux aussi incerlains sont justiciables des sejours dans les villes d'hiver (Boyer, 1982: 140).

\section{Animation}

Vingt ans plus tard, terminant une période d'enseignement, j'allai travailler avec une équipe d'action sociale en milieu ouvrier. Mais avant cela, durant les vacances, j'en profitai pour rejoindre des camps d"animation en milieu populaire auxquels $\mathrm{j}$ "avais participé pendant les étés 1956 et 1957.

A mon grand étonnement, c'est au littoral français, sur lạ Côte d'Opale, au Nord, que les organisateurs attendaient cette fois les volontaires, en 1960. En fạit, à part des vacances estivales avee mes parents à Westende-Bains en 1937, au début des congés payés je n'avais jamais été à la mer en saison touristique. Je n'en avais gardé d'ailleurs que le mauvais souvenir de sévères et répétés coups de soleil avec fiêvre, médecin et journées alitées. Faisant partie du scoutisme, ce groupe fuyait au contraire les plages très fréquentées et privilégiait plutôt les forêts et les riviêres du Sud du pays pour les camps et les excursions : nature, plein air, à l'écart des foules !

Voilà que je me retrouvais de plain-pied au cour d'un phénomène de société : le tourisme de MASSE ! En effet, apres la guerre mondiale de 1940-1945, les familles et les jeunes ont envahi par millions les littoraux de toute l'Europe, du Nord et, de plus en plus, du Sud. Les hôtels inaccessibles financièrement aux touristes à revenus modestes, les vacanciers ont préféré la tente et le camping. Par ailleurs, la voiture a provoqué la dispersion, loin et en dehors des stations touristiques historiques, sur l'ensemble des côtes, et prive par contre les campeurs des services que villes et villages offraient habituellement à ces derniers.

La mer, le soleil, la nature constituaient un véritable ANTIDOTE aux miasmes des concentrations urbaines et industrielles, au stress 
et aux contraintes du travail ou des études. A noter que le tourisme balnéaire a, jusqu' à présent d ailleurs, toujours obtenu la préférence parmi les destinations proposées par le marché, avec des majorités de $60 \%$ à $70 \%$.

Donc, chacune des équipes, mixtes, composées d une vingtaine de jeunes, étudiants ou déjà travailleurs, majoritairement Français et issus des mouvements de jeunesse, fut envoyé avec deux animateurs, trois tentes, tables et bancs dans un des grands campings de 4000 à 6000 personnes de ce chapelet de villages de toile. A part peut-être une expétience de moniteur de colonie de vacances, personne n'était préparé à exercer une telle animation, tout à fait inédite, il y à quarante ans ! Tout était à inventer face à une foule inorganisé et anonyme, tegroupee dans un ghetto qui ne disposait d'aucun service collectif sinon des toilettes et des lavabos, éventuellement d'un magasin-caférestaurant et, quelquefois, d'une nesse dominicale celebrece par un prêtre venant de l'extérieur, commerce oblige!

D'où, premier objectif, partir à la decouverte de la jeunesse du camping : qui étaient-ils ? D'où venaient-ils ? Etaient-ils avec leurs parents, seuls ou avec des copains ? Est-ce qu'ils étudiaient ? et quoi ? Ou travaillaient-ils dejà ? et en quoi ? Quelles activites de loisirs pratiquaient-ils ici et chez eux ? Où se rassemblaientils éventuellement?

Face à des centaines de jeunes vacanciers, la plupart accompagnant leurs parents, à cette époque encore, coupés de leurs relations amicales nouées dans la vic quotidienne de leur lieu d'origine, notre bande mixte et dynamique, ouverte et amusante, a constitué un véritable aimant qui attirait les vacanciers par sa charge affective et liberatrice.

Le volley-ball sur la plage, les soireses à la discothèue, la participation aux fêtes populaires et aux campagnes publicitaires... ont permis aux membres de l'équipe de crét des liens avec bon nombre de garçons et de filles de leur âge. Le point de convergence ? Le CAFË que nous prenions à quatorze heures, à lä fin de notre repas, a notre tente, et anquel toutes nos nouvelles connaissances étaient invitées; quelle ambiance de chansons, de partages et de débats ! C'est à ce carrefour, comme à l'oasis et au puits dans le désert, que se formulaient les attentes comme aussi des suggestions et des propositions susceptibles d'y répondre. Que de compétences présentes dans le camping et rassemblées lầ, pretes à s" engager : animateurs sportifs, dessinateurs, chanteurs, mimes, guitaristes, dactylos, débatteurs, animateurs de soirées ou de ciné-clubs, militants de la JOC ou des jeunesses communistes, tous volontaires pour constituer le comité $\mathrm{d}^{\dagger}$ animation. Après quatre jours seulement, car il ne restait que deux semaines avant le départ du 31 juillet, un programme d'activités avait éte dresse precisant lieux et horaires, en plusieurs langues, dactylographié et imprimé au a stencil s durant la nuit, distribué aux lavabos et collé sur la porte des toilettes, diffusé au marche, sur la plage et dans les lieux de rassemblements populaires. Les plaques d'immatriculation des voitures ainsi que la langue émise par les radios portatives... représentaient autant de possibilites d'entrer en contact, sans problime, avec des citoyens de divers départements et pays d'origine. Les fêtes nationales - le 14 juillet pour les Français et le 21 juillet pour les Belges - ont été 1'occasion de participer ou même de mettre sur pied feux de camp ou soirées de cabaret de grande qualite artistique. A la fin du spectacle, très convivial et participatif, l"animateur mettait en évidence, à l'occasion des remerciements, l'incroyable mobilisation de bonnes volontés et de talents, de pays et de langues differentes, comprenant enfants et jeunes, adultes et seniors, ayant contribué à creer de la beauté et du rire, de l'espoir et une fraternisation internationale, prémices d'un monde nouveau et meilleur.

Après la mer du Nord, durant les deux saisons suivantes, 1961 1962, nous nous sommes tournés vers le département du Var, sur la Mediterranée. Il était temps de commencer nos activités dans notre pays, sur la côte belge en 1963, a La Panne, unique avec ses dunes de sables de trois kilomètres de large ! Il fallait relever un nouveau defi ! En effet, notre littoral de soixante-dix kilomètres de long se caractérise par de grosses stations touristiques de type urbain offrant, outre des hôtels et des campings, une majorite, à $75 \%$, de locations d'appartements de vacances par le biais d'agences immobilières ad hoc. A La Panne, les mois de juillet et d'août concentraient respectivement, durant deux b trois semaines, pres de 100000 estivants, dont 25000 enfants et jeunes, alors que le nombre d' habitants autochtones s'elevait a 3000 seulement ! Aucun loisir planifié, sinon des locations dé vélos pour les enfants, sept discothèques pour les jeunes, trois cinémas, des campagnes publicitaires et des bals populaires organisés par des firmes commerciales : pas de bibliotheque publique ouverte ni de salle communale disponible ; aucun sport collectif organisé sur la plage ! ! !

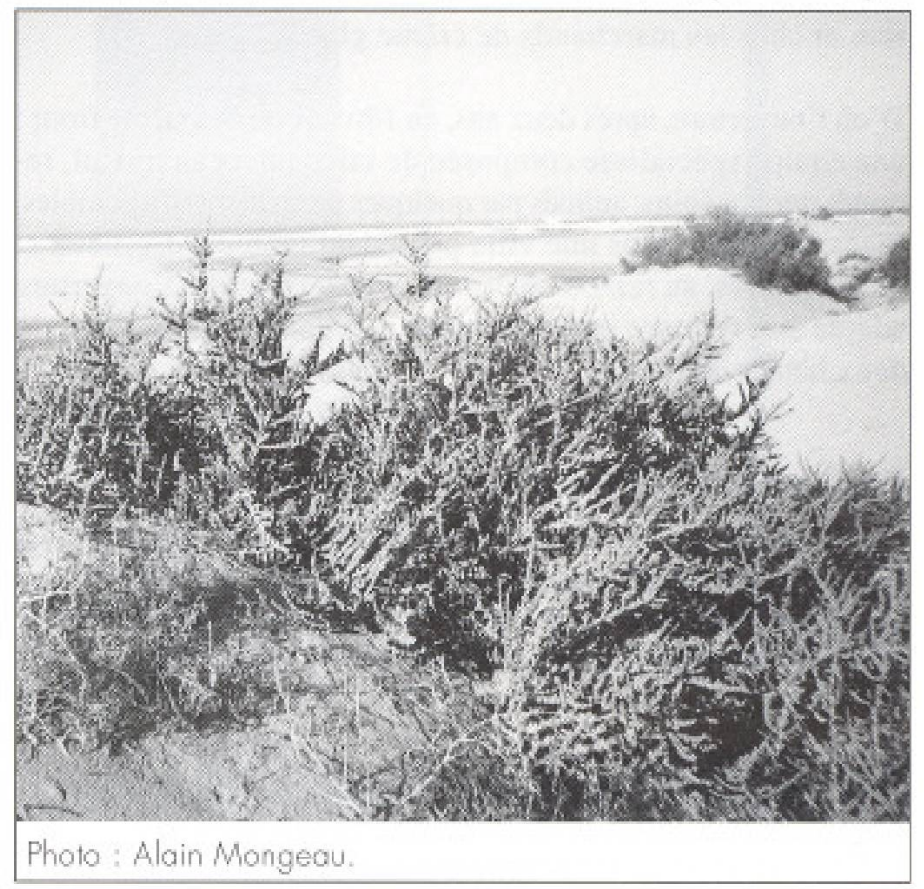


En consequence, cette \& surconcentration * d'un million de touristes durant chacun des deux mois de saison a provoqué une surcharge de population difficilement supportable par l'environnement : dunes aplanies et occupées par des campings et progressivement par des villas, bord de mer bâti d'immeubles à dix étages formant un front de bêton inesthétique et occasionnant des courants d'air désagréables, eaux usées et matières fécales rejetées à marée haute en mer. Vu l'absence pendant vingt ans de stations d'épuration adaptées à un tel volume de déjection, déchets abandonnés sur la plage la transformant en poubelle publique.

À l'époque, des journalistes ont lancé un cri d'alarme tellement la situation était devenue critique et ils ont déclenché des actions de sensibilisation destinées au grand public comme aux responsables politiques, par exemple : Louis Bériot (1976) a cré la Fondation Espace pour demain et Marcel Clébant (1973) l'Opération mondiale message â la mer.

Cela explique que l'affluence de consommateurs touristiques ait nécessité l'embauche d'un nombre important de saisonniers, majoritairement étudiants, venant de l'extérieur, pour suppléer à la carence de main-d' ceuvre locale. D'où, une exploitation éhontée du personnel, sans contrat légal ni protection sociale : longues heures de prestation, sept jours sur sept, sans congé de récupération et à des salaires de misère ! Faible syndicalisation et rejet des étudiants considérés comme des enfants de bourgeois, futurs cadres professionnels, médecins ou avocats, donc ennemis de classe!

Les membres de l'equipe d'animation, outre les initiatives en matière de loisirs, ont très vite fraternisé avec les autres jeunes - surmenés, isolés, exploités - au travail dans les cafés, les restaurants, les hôtels, les crêperies, les magasins d'alimentation et chez les marchands de creme glacé...

D'où l'ouverture, après deux ans, en 1965, d'un deuxième front : une équipe spécialisée composée de saisonniers au travail, recrutés par nos soins, animés par quelques permanents disponibles, logés ensemble dans une villa possédant un local d'accueil et de rencontres, au rez-de-chaussée, avec un horaire d'ouverture adapté aux rythmes professionnels, notamment le soir jusqu'à deux heures du matin.

\section{Professionnalisation}

Les évaluations successives ont engrangé des constatations dont il fallait chercher les causes et les remèdes, dégager une méthodologie et une stratégie d'animation sociale, culturelle et finalement sociopolitique. D'association de fait, les camps d'animation et les équipes d'étudiants-travailleurs, après six ans, se sont mués, en 1969, en association sans but lucratif, CultureTourisme-Loisirs (CTL), dont le signataire, fondateur et accompagnateur de l'expérience depuis ses débuts, est devenu le secrétaire permanent et le coordonnateur.
Parmi un ensemble d' améliorations progressives au départ d'une situation intolérable ${ }^{3}$, le CTL a été un acteur dynamique et un interpellateur, tant au plan de la recherche qu' à celui des pouvoirs politiques (Hut, $1991: 9-23)^{4}$. Depuis 1970, les équipes d'action sur le terrain ont procédé à une $\alpha$ analyse globalle * des stations touristiques (La Panne, LaRoche, Dinant...) comprenant les aspects interdépendants aussi bien environnementaux qu'économiques, sociaux, culturels et politiques. Comment expliquer la s réduction s unidimensionnelle du tourisme à l'exclusive exploitation commerciale ?Quel(s) rôle(s) jouaient, sur ce plan, les mandataires politiques communaux? La divulgation, par tracts, débats et articles de presse, de l'accumulation entre les mêmes mains et les mêmes personnes des pouvoirs économiques (patrons et organisations professionnelles), culturels (comité des fêtes) et politiques (syndicat d'initiative) a provoqué la * répression * par ces derniers vis-ă-vis des équipes d'animation et de travailleurs saisonniers et même leur * expulsion * de campings et de locations touristiques ainsi que le lock-out de tout le patronat commerçant et touristique de la cồte!

Par ailleurs, des voyages d'études sur les littoraux du pourtour méditerranéen, de 1970 à 1977 , ont permis de découvrir les pratiques d'autogestion suivant l'option culturelle et politique que le CTL s'était définie:

- sur l'expérience autogestionnaire demeurée vivace, en Algérie, après son indépendance, particulièrement dans l'hôtellerie et les clubs de vacances côtiers de Moretti, de Sidi-Feruch et de Tipaza :

- en Yougoslavie, l'exercice de l'autogestion, étatiquement institutionnalisée, intégrant dans la politique communale la gestion économique, sociale et culturelle des hôtels et des campings des centres touristiques de Zadar, de Trogir et de Dubrovnik, faisant participer d'ailleurs les saisonniers aux assemblées délibératives, en fin de saison, dont le but était de répartir les bénéfices de l'exploitation, y compris pour la formation et la recherche d'embauche du personnel pour une deuxième saison dans le tourisme d'hiver, en Allemagne *

- enfin, dans le cadre de l'animation des centres de vacances d'une organisation de tourisme social pour jeunes, la Sotutour en Tunisie, sur les plages d'Hammamet, de Sfax ou dans l'île de Kerkéna.

La réflexion, après saison, sur les actions socioculturelles menées depuis 1963 par les équipes d'animateurs du CTL, dans les stations touristiques du littoral et des Ardennes, parmi et avec les campeurs et les locataires de meublés, aboutit notamment à lá constatation d'un * blocage * culturel. En effet, les travailleurs à revenus modestes qui partaient en vacances durant leurs congés payés ont avoué, lors des échanges, des débats et des sondages, qu'ils ne voyaient pas d'autres * alternatives * que le camping ou le meublé touristique. En fait, ils se trouvaient pieds 
et poings liés, â la merei des conditions de location imposées d'une manière unilatérale, avec un contrat léonin et non réglementé officiellement, par les patrons de campings et d'agences de location touristique.

C'est pourquoi le CTL a créé en son sein, en 1973, un Centre de Recherche et de Formation en Animation Touristique (CERFAT), afin de programmer études et matériaux pédagogiques sur « l'image des vacances * véhiculée par les médias : affiches et publicités touristiques, livres pour enfants et bandes dessinés... Quelle a idéologie $*$ des loisirs, des vacances et du tourisme se dégagerait des messages publicitaires directs ou indirects diffusés par les industries du tourisme et de la culture ? Le prestige des classes privilégiées n'imposerait-il pas, là aussi. aux classes populaires un \& modèle \& de comportement touristique pratiqué antérieurement par les premières et que les dernières s'empresseraient à leur tour d'imiter plus ou moins ?

\section{Structuration}

Depuis lors et jusqu'à présent, la \& surconcentration s d'estivants, regroupés dans le même endroit en même temps, a motivé la recherche et les engagements du CTL et de son fondateur.

Les vacanciers, d'abord. Pourquoi une attirance aussi prépondérante pour cette destination, la mer ? Serait-ce en raison des conditionnements devenus insupportables de la vie scolaire et professionnelle, de la pollution et du stress du milieu urbain ? Du besoin de l'homme cybernétique de retrouver l'immensité et l'horizontalité de l'océan pour y reposer ses yeux et son esprit ? Face à une mort inéluctable, le désir d'éternité que lui rappelle celle qui a participé à la naissance du monde, qui l'attend, fïdèle au rendez-vous, et lui survivre, matrice de vie et de renouveau?

L'histoire des religions a mis en évidence, à juste titre, la permanence, dans l'inconscient personnel et collectif, des archétypes mentaux ainsi que du symbolisme et des rites liés à l'eau et à la mer, depuis les hommes dits primitifs jusqu'à ceux qui se réclament de la modernité5. L'étude des messages publicitaires, réalisée par le CERFAT, n'a-t-elle pas démontré à quel point leurs concepteurs utilisent les données de la psychologie des profondeurs pour manipuler les acheteurs potentiels : un couple, nu et innocent, sur le bord d'un rivage exotique et paradisiaque !

A souligner aussi, l'agression de l'espace maritime par la surcharge des populations vacancières et des equipements touristiques dont la démesure appelle des décisions politiques urgentes et radicales afin de protéger ces lieux uniques, fragiles et si indispensables à l'équilibre de l'écosystème, y compris des humains qui constituent un des maillons de la chaine du vivant.

Le CTL a été la première association francophone à dénoncer, en 1970, la nocivité mortifère d'un vaste projet immobilier que des promoteurs, soutenus par la municipalité, voulaient implanter au coeur du dernier et remarquable échantillon des dunes côtières, à La Panne.

D'où l'intérêt permanent et la collaboration active, en solidarité, du CTL avec les associations qui luttent pour la protection de l'environnement, et sa participation aux campagnes des organisations internationales, du Conseil de l'Europe et des Nations Unies pour la défense et les * soins intensifs \& en faveur des littoraux menacés, d'Europe et des autres continents ${ }^{6}$.

Enfin, la cause de cette paradoxale descente aux enfers en réponse à l'offre paradisiaque promise par les opérateurs touristiques, c'est la simultanéité de la présence vacancière, condamnée à prendre ses congés scolaires et professionnels dans le cadre étroit et obligatoire du calendrier légalement imposé par les pouvoirs politiques et économiques de la société.

L'étalement des vacances représente la solution qui s'impose impérativement et d'urgence, malgré sa complexité et l'interdépendance de ses diverses composantes. Le Bureau International du Tourisme Social (BITS), dont le CERFAT est membre depuis 1972 , lutte sur tous les fronts pour faire admettre la pertinence de cette alternative et permettre sa réalisation concrète. Mais son application ne s'oppose pas seulement aux résistances des intérêts immédiats des entreprises, de l'organisation traditionnelle des rythmes scolaires et d'une majorité d'enseignants conservateurs, des habitudes (et des moyens) de ceux qui ne sont pas encore ou plus dans la * vie active *, du stéréotype de 1 'excellence des mois de juillet et d'août... Ne remet-elle pas en cause une soi-disant "neutralité " du temps ? Ne révèle-t-elle pas à l'cuvre une logique souterraine, structurelle et synergique : $*$ Le Temps, e'est de l'Argent ! s ?

En conséquence, la CERFAT organise, dès 1977, un cycle de rencontres intitulées \& L'aménagement du temps, une mystification? ? avec des représentants d'organisations syndicales, socioculturelles, educatives, scolaires, touristiques et sociopolitiques... pour s'interroger sur les options justifiant, à l'époque, les réductions ou non du temps de travail, les intentions implicites du patronat proposant les horaires variables ou décalés, les enjeux de l'étalement des vacances et des scénarios d'aménagement du temps. D'où la nécessité d'une « ré-appropriation idéologique et concrète du temps $*$ par les travailleurs, les citoyens, les usagers des temps libres w (Hut, 1991: 12-13).

Voilà les axes prioritaires qui ont marqué ma vie depuis le choc frontal de 1960 : défense et réaménagement de l'espace, notamment balnéaire, dans une perspective de développement durable ; réappropriation du temps, où interviennent tous les aspects de la vie personnelle et collective ; recréation d'une philosophie des loisirs et d'un mode de vie liberateur des vacanciers et des personnes à leur service, au nom d'une éthique qui interpelle la pratique, l'industrie et les politiques touristiques. 


\section{Ressourcement}

La boucle est bouclée ! Désormais, ma demande de mise à la retraite acceptée, je suis libéré des contraintes d'un emploi du temps, professionnel ou volontaire, tributaire des autres. J'ai le choix d'organiser ma vie selon l"horaire que je souhaite, compte tenu cependant des précautions dues à ma santé comme aussi des limites financières d'un pécule assez modeste de retraité. Allergique et cardiaque, c'est la mer qui me convient le mieux. Peut-être des séjours en thalassothérapie, comme à sept ans. Mais surtout, je puis bénéficier tant des moments les plus favorables de la météo prévue que des conditions touristiques les plus avantageuses!!!

Décider d'une journée d'excursion, à la place d'Ostende, soleil annoncé, au tarif ferroviaire préférentiel de $100 \mathrm{FB}$ aller-retour accordé aux plus de soixante-cinq ans, un cadeau ! Prendre un mois de repos au littoral, en appartement touristique, en basse saison, au prix de location le moins cher, agrémenté d'un taux d'iode élevé et de la température la plus clémente du pays. microclimat de La Panne oblige!

Partir en vacances, au moindre coût en arrière-saison, sur la côte landaise, à l'ensoleillement agréablement tempéré, aux eaux de baignade d'une douceur méridionale !

Retrouver les amis du Québec, en tarif d'avant-saison en juin, évitant les chaleurs trop fortes et déconseillées médicalement de l'été, rejoindre mes lectures d'enfance, aux images faussées de " méchants indiens s et les rectifier en participant à nouveau au Festival des Autochtones, à Montréal, organisé par l'Association Terre en Vues ${ }^{7}$, où je rencontrerai de vrais Amérindiens ! Pour terminer, un vieux rêve caressé depuis les vingt-trois ans que je viens au Québec : descendre le Saint-Laurent en bateau de croisière et séjourner dans les îles atlantiques et océanes de la Madeleine et du parc naturel d'Anticosti ! En service de presse, peut-être ? Chiche !

\section{Notes}

1 Corbin développe, en plus de la dimension médicale, la dimension imaginaire et psychologique, sensorielle et ludique de la fréquentation des rivages (Boyer, 1982).

2 Voir : Corvol, 1958 et Augier, 1981.

3 Lire à ce sujet le numéro Téoros sur le Bas Saint-Laurent, 13 (1). mars 1994, dont l'article de André Hut, * Redécouvrir l'intérieur *. p. 46-48. Lire aussi : La Côte belge, Le guide, Éditions La Renaissance du Livre, Tournai, 2000. Mer du Nord, un art de vivre entre plages et polders, texte de Brigitte Forgeur et photos de Christian Sarrarnon, même édileur et année, collection L'Esprit des lieux. $\mathrm{La}$ Cote belge et son arrière-pays, édite en 2000 par Westloerisme, Kasteel Tillegem, B.8200 Saint-Michiels (télécopieur : 32.50.38.02.92), gratuit, Fédération du Tourisme de la province de Flandre-Occidentale.

4 L'introduction décrit les actions sur le terrain et la recherche du CTL. sur divers fronts de lutte, à partir de 1970.
5 Lire le chapitre $\mathrm{V}$ : Les eaux et le symbolisme aquatique du Traite d'histoire des religions, de Mircea Eliarde, 1964/1975.

6 Lire le numéro de Téros sur Tourisme et environnement, 10 (1). mars 1991, dont les articles de André Hut, p. 33 : Le tourisme patrimonial : l'expérience belge, et p. 43 : Tourisme et environnement : des actions internationales.

7 Terre en Vues, Société pour la diffusion de la culture autochtone. 6865, rue Christophe-Colomb, bureau 102, Montréal (Quebec), H2S $2 \mathrm{H3}$; téléphone : (514) 278-4040 ; télécopieur : (514) 278-4224.Bibliographie

\section{Bibliographie}

ARTEA Conseil (1990), La her ef son univers, conception-realisation ARTEA Conseil, Centre de la Mer et des Eaux, 195, rue St-Jacques, 75005 Paris ; téléphone : 33.1 .44 .32 .10 .70 (Musées, centres de la mer, aquarums, espaces naturels, organismes, associations, centres de documenLation).

Augier, Paul (1981), Quand les grands dacs valsaient a Nice, Collection Quand, Editions Arthème Fayard, Paris.

Bériot, Louis (1976), Les pleds dans la mer, Editions Jean-Claude Lattès. Paris.

Boyer, Mare (1972 et 1982), Le tourisme, Collection Peuple et Culture, Editions du Seuil, Paris, 261 p.

Clébant, Marcel (1973), Croisade pour la mer, Éditions Stock, Paris. Corbin, Alain (1988), Le territoire du vide, rOccident et le désir du rivage, 1750-1840, Collection Champs, Flammarion, Paris, 411 p.

Corvol, Robert (1958), La Côte d'Azur à la Belle Épogue, Éditions Arthème Fayard, Paris.

Courrier de l'Unesco (1998), * 20000 mondes sous les mers w, dossier thématique du Courrier de l'Unesco, Paris (La mer et ses trésors, menaces et conflits, des lois pour l'océan).

Courrier de 1'Unesco (1991), a Dialogue avec la mer *, dossier du Courrier de l'Unesco, Paris (iconographie, les dieux et le gouffre, les voix de la mère, l'autre coté du miroir, la maison bleue, le * Plan bleu $\%$ pour l'avenir de la Méditerranée).

Désert, Gabriel (1983), La vie quotidienne sur les plages normandes du second empire aux folles années, Collection La vie quotidienne, Editions Hachette, Paris.

Eliarde, Mircea (1964/1975). Traité d'histoire des religions, collection Petite Bibliothèque Payot, n 312.

Gếo (1996), \& L'Atlantique s, numéro thématique de Géo, ñ" 210. Paris (Les îles du grand large, le guide du littoral français, liste de musées en France et à l'ếtranger).

Hut, André (1991), * Congés payés $1936 \%$, Histoire et ldéologies, collection Reflet, Éditions Artel, Bruxelles.

Hut, Andre, Boyer, Marc, et Mucci, Aimé (1985), * Le tourisme, toute une histoire s., Téoros, 14 (2).

Naturopa, Conseil de l'Europe (1998), Les espaces côtiers : pour une gestion durable, dossier spécial, no 88 de Naturopa, Conseil de l'Europe (Très intéressant. F67075, Strasbourg ; téléphone : 33.3.88,41 22.78 ; télécopieur : $33.3 .88 .41 .27,15$ ). 